\title{
Viabilidade de sementes de feijão caupi após o tratamento com óleo essencial de citronela (Cymbopogon winterianus Jowitt)
}

\author{
XAVIER, M.V.A.; OLIVEIRA, C.R.F.*; BRITO, S.S.S.; MATOS, C.H.C.; PINTO, M.A.D.S.C. \\ Unidade Acadêmica de Serra Talhada, Universidade Federal Rural de Pernambuco, (UASTIUFRPE). Fazenda \\ Saco, s/n, Caixa Postal 63, CEP: 56900-000, Serra Talhada-Brasil *crfoliveira@hotmail.com
}

\begin{abstract}
RESUMO: O tratamento de sementes com óleos essenciais é um método alternativo que auxilia o manejo integrado de pragas. O objetivo deste trabalho foi avaliar a influência do tratamento de sementes de feijão Vigna unguiculata (L.) Walp. com o óleo essencial de citronela (Cymbopogon winterianus Jowitt). Foi avaliado o efeito do óleo essencial de $C$. winterianus sobre $V$. unguiculata nas dosagens $20 \mu \mathrm{L}, 15 \mu \mathrm{L}, 10 \mu \mathrm{L}, 5 \mu \mathrm{L}$ e testemunha. A qualidade fisiológica das sementes foi determinada pela porcentagem de emergência, velocidade de emergência e índice de velocidade de emergência. A análise dos dados foi realizada no delineamento inteiramente casualizado, disposto em esquema fatorial $5 \times 2$ com cinco repetições. As sementes fumigadas apresentaram diferenças estatísticas entre os parâmetros avaliados em relação à testemunha. O óleo essencial de citronela revelou potencialidade alelopática sobre a germinação de sementes de feijão que variou de acordo com a concentração do óleo.
\end{abstract}

Palavras-chave: Germinação, Vigna unguiculata, Cymbopogon winterianus, potencial alelopático, óleo essencial

\begin{abstract}
Viability of Vigna unguiculata bean seeds treated with essential oil of Java grass (Cymbopogon winterianus Jowitt). Seed treatment with essential oils is an alternative method tool in integrated pest management. The objective of this study was to evaluate the effect of treating Vigna unguiculata (L.) Walp. bean seeds with essential oil of Java grass (Cymbopogon winterianus Jowitt). The effect of $C$. winterianus essential oil on $P$. vulgaris was evaluated at levels of $20 \mu \mathrm{L}, 15 \mu \mathrm{L}, 10 \mu \mathrm{L}, 5 \mu \mathrm{L}$ and control. The physiologic quality of the seeds was determined by percentage emergence, speed emergence and speed emergence index. The data analysis was carried out using an entirely randomized design, in a $5 \times 2$ factorial scheme with five repetitions. The fumigated bean seeds showed the statistics differences among the analyzed parameters when was compared with the no treated check. The essential oil of Java grass revealed allelopathic potentiality on bean seed germination which varied according to the oil concentration.
\end{abstract}

Key words: Germination, Vigna unguiculata, Cymbopogon winterianus, allellopathic potential, essential oil

\section{INTRODUÇÃO}

A citronela (Cymbopogon winterianus Jowiit) é espécie medicinal e aromática, pertencente à família Poaceae, cujo cultivo no Brasil tem crescido devido à procura pelo seu óleo essencial. Conhecida por fornecer óleo essencial para fabricação de repelentes contra mosquitos, o óleo essencial de citronela contém muitos componentes e é rico em citronelal e geraniol (Guenther, 1949; Rocha et al., 2000; Menezes, 2005; Furtado et al., 2005).

No Brasil e em outros países da América Latina, o feijão caupi é considerado alimento básico e um dos mais consumidos, por ser fonte de fácil acesso à proteínas, vitaminas e minerais, além de possuir elevado conteúdo energético (Oliveira \& Carvalho, 1988; Guzmán-Maldonado et al., 1996).

Os estudos sobre o potencial das espécies vegetais visando obter óleos essenciais como métodos de controle de patógenos e pragas no tratamento de sementes vêm crescendo, como os inseticidas botânicos, pós, extratos botânicos e óleos essenciais de origem vegetal (Shaaya et al., 1997).

Os óleos essenciais geralmente são

Recebido para publicação: setembro de 2011

Aceito para publicação: março de 2012

Rev. Bras. PI. Med., Botucatu, v.14, n.esp., p.250-254, 2012. 
utilizados como um método de controle eficaz, que visa à redução dos custos, menor risco por intoxicação do ambiente e dos alimentos por contaminação química. Algumas pesquisas mostram que o tratamento com óleo de Glycine max (L.) Merr. em sementes armazenadas de Phaseolus vulgaris L. não influenciou na germinação e nem na viabilidade das sementes, indicando que este pode ser utilizado no controle das pragas de grãos armazenados não inviabilizando a germinação das sementes (Kéita et al., 2001; Roel, 2001).

Tanto o armazenamento como o tratamento de sementes tornam-se práticas de extrema importância para a manutenção da germinação e do vigor das sementes, contribuindo para a produtividade satisfatória (Machado, 2000). No entanto, nas regiões tropicais, o armazenamento é uma das principais limitações encontradas devido à influência de diversos fatores na manutenção da viabilidade das sementes, como qualidade fisiológica inicial da semente, condições climáticas durante a maturação, grau de umidade, temperatura de armazenamento e principalmente ação de microrganismos e insetos (Carvalho \& Nakagawa, 2000).

Acredita-se que o Brasil, por apresentar grande riqueza e abundância em termos de espécies vegetais, pode ser considerado um grande impulsionador das pesquisas acerca do emprego de óleos essenciais para o tratamento de sementes, bem como para a descoberta de efeitos inseticidas de espécies vegetais antes exploradas para outros fins, mas que seus subprodutos podem ser aproveitados para o controle de pragas em diversas formas e patógenos que inviabilizem a comercialização e uso de sementes para fins comerciais.

Lopes et al. (2000) observaram que produtos naturais à base de frutos de pimenta do reino moída são eficientes controladores de insetos pragas de feijão caupi armazenado e o uso não afetou a qualidade física e fisiológica destas sementes.

Assim, o presente trabalho foi realizado com o objetivo de verificar os efeitos do óleo essencial de citronela (C. winterianus), em diferentes dosagens sobre o potencial fisiológico das sementes de feijão caupi tratadas por meio do processo de fumigação e impregnação.

\section{MATERIAL E MÉTODO}

O presente trabalho foi desenvolvido na Universidade Federal Rural de Pernambuco, Unidade Acadêmica de Serra Talhada, em condições de viveiro, temperatura ambiente, município Serra Talhada - PE. Foram utilizadas sementes da cultivar BRS Guariba tratadas com o óleo essencial de $C$. winterianus, obtido da empresa Quinarí Fragrâncias e Cosméticos LTDA. As dosagens utilizadas foram $20 \mu \mathrm{L}, 15 \mu \mathrm{L}$,
$10 \mu \mathrm{L}$ e $5 \mu \mathrm{L}$ e testemunha. Também foram utilizadas duas formas de aplicação do óleo, aplicação direta e indireta. Na primeira forma de aplicação as sementes foram diretamente impregnadas com o óleo essencial no interior de recipiente plástico, com o auxílio de pipetador automático, mediante agitação manual durante dois minutos. No segundo método de aplicação utilizou-se câmaras de fumigação (Adaptado de Aslan et al., 2004), compostas de recipientes de vidro onde as sementes não tiveram contato direto com o óleo. Neste caso utilizou-se pipetador automático para a impregnação do óleo em tiras de papel de filtro de $5 \times 2 \mathrm{~cm}$, as quais foram fixadas na superfície inferior da tampa dos recipientes.

A semeadura foi executada 24 horas depois do tratamento das sementes com o óleo, sendo utilizadas bandejas de isopor com 200 cédulas e o substrato para plantas Tropstrato HA para hortaliças. Utilizou-se uma semente por cédula, na profundidade de aproximadamente $3 \mathrm{~cm}$, realizando-se irrigações diárias.

Foi empregado o delineamento inteiramente casualizado, em esquema fatorial $5 \times 2$ (cinco dosagens do óleo $x$ duas aplicações), com cinco repetições de 20 sementes cada. A partir do segundo dia após a implantação do experimento, até o 8 dia, realizou-se a contagem diária das plântulas emersas. Com isto, obteve-se a Porcentagem de emergência (\% E), a Velocidade de emergência (VE), o Índice de velocidade de emergência (IVE) e o Coeficiente de velocidade de emergência (CVE), empregando-se as seguintes fórmulas:

a) Velocidade de emergência (Edmond \& Drepala, 1958):

$$
V E=\frac{\left(N_{1} E_{1}\right)+\left(N_{2} E_{2}\right)+\ldots+\left(N_{n} E_{n}\right)}{E_{1}+E_{2}+\ldots+E_{n}}
$$

Onde: VE = velocidade de emergência (dias); $\mathrm{E}=$ número de plântulas emergidas em cada repetição; $\mathrm{N}=$ número de dias da semana a cada contagem.

b) Índice de velocidade de emergência (Maguire, 1962):

$$
\text { IVE }=\underset{----}{\mathrm{N}_{1}}+\frac{\mathrm{E}_{2}}{\mathrm{~N}_{2}}+\ldots+{ }_{---}^{\mathrm{N}_{\mathrm{n}}}
$$

Onde: IVE = Índice de velocidade de emergência; $E$ = número de plântulas emergidas em cada repetição; $\mathrm{N}$ = número de dias da semana a cada contagem.

c) Coeficiente de velocidade de emergência (Furbeck et al., 1993):

$$
C V E=\frac{E_{1}+E_{2}+\ldots+E_{n}}{\left(N_{1} E_{1}\right)+\left(N_{2} E_{2}\right)+\ldots+\left(N_{n} E_{n}\right)} \times 100
$$


Onde: $\mathrm{CVE}=$ Coeficiente de velocidade de emergência; $E=$ número de plântulas emergidas em cada repetição; $\mathrm{N}=$ número de dias da semana a cada contagem.

Os resultados foram submetidos à análise de variância, sendo as médias comparadas pelo teste de Tukey ao nível de $5 \%$ de probabilidade (Canteri et al., 2001).

\section{RESULTADO E DISCUSSÃO}

Verificou-se que não houve diferenças significativas para a porcentagem de germinação de sementes de $V$. unguiculata tratadas com o óleo essencial de $C$. winterianus em diferentes dosagens. Entretanto, quando comparadas à testemunha, sementes não-tratadas apresentaram maior porcentagem de germinação (Figura 1).

Em relação ao tipo de aplicação do óleo essencial (fumigação ou impregnação) também não foram observadas diferenças estatísticas para os dois métodos (Tabela 1). Entretanto, a porcentagem de germinação foi superior na ausência do óleo essencial de citronela, sendo, portanto, um indicativo da ação inibitória deste.

Dudai et al. (1999), trabalhando com óleos essenciais das plantas Origanum syriacum, Micromeria fruticosa e Cymbopogon citratus, observaram atividade inibitória, em baixas concentrações destes óleos, sobre a germinação de Amaranthus blitoides, Amaranthus palmeri, Euphorbia hirta L., Sinaps nigra, Trifolium campestri e Lycopersicum esculentum L.

Lobato et al. (2007) mostraram que a ação do óleo essencial de Pipper aducum no tratamento de sementes de $V$. unguiculata não apresentou respostas fitotóxicas sobre a germinação, em altas concentrações do óleo.

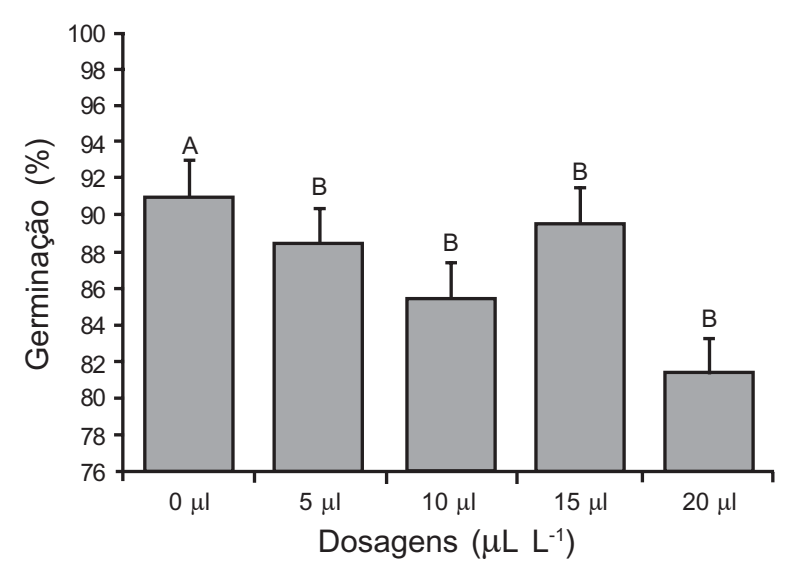

FIGURA 1. Efeito de diferentes dosagens do óleo essencial de $C$. winterianus sobre a germinação (emergência) de sementes de $V$. unguiculata.
TABELA 1. Porcentagem de germinação (emergência) de sementes de Vigna unguiculata submetidas ao óleo essencial de Cymbopogon winterianus, sob diferentes aplicações.

\begin{tabular}{lc}
\hline Método de Aplicação & Germinação \\
\hline Fumigação & 88,75 B \\
Impregnação & $83,25 \mathrm{~B}$ \\
Testemunha & $91,00 \mathrm{~A}$ \\
\hline
\end{tabular}

*Médias seguidas de mesma letra, nas colunas, não diferem entre si significativamente pelo teste de Tukey, a $5 \%$ de probabilidade.

De acordo com Medeiros et al. (2007), o uso de folhas de nim também não mostrou efeitos tóxicos para as sementes de caupi em relação à porcentagem de germinação, exceto para massa seca das plântulas.

Foi observado que as sementes não-tratadas (testemunha) em relação aos dois métodos de aplicação do óleo foram as que obtiveram o maior Índice de Velocidade de Emergência, sendo observadas diferenças estatísticas entre a testemunha e as maiores dosagens $(10 \mu \mathrm{L}, 15 \mu \mathrm{L}$ e $20 \mu \mathrm{L}$ ) quando o óleo foi fumigado, e diferenças entre a testemunha e as menores dosagens $(5 \mu \mathrm{L}$ e $10 \mu \mathrm{L})$ quando o óleo foi impregnado (Tabela 2).

Para o parâmetro Velocidade de Germinação observou-se os maiores valores para as sementes tratadas, não importando o método de aplicação do óleo essencial (fumigação ou impregnação). Neste caso, houve diferenças estatísticas apenas entre a testemunha e todas as dosagens utilizadas, nos dois métodos (Tabela 2). Assim, pode-se inferir que o óleo essencial de citronela foi prejudicial ao vigor das sementes de feijão tratadas.

No que se refere ao Coeficiente de velocidade de emergência, todos os tratamentos não diferiram significativamente entre si, mas diferiram da testemunha, sendo esta a que apresentou os maiores valores para os dois métodos de aplicação do óleo, demonstrando mais uma vez o efeito deletério do óleo essencial de citronela no potencial fisiológico das sementes de caupi (Tabela 2). Como o citronelal e o geraniol são os compostos majoritários do óleo essencial de citronela, e sendo monoterpenos, provavelmente são os responsáveis pela inibição da germinação das sementes testadas.

Dessa forma, os dados revelaram que o uso de diferentes dosagens do óleo de citronela interferiu de forma negativa no vigor das sementes, não sendo, portanto adequado o tratamento de sementes com o referido óleo, nas dosagens avaliadas. 
TABELA 2. Médias estimadas da velocidade de emergência (VE), índice de velocidade de emergência (IVE) e coeficiente de velocidade de emergência (CVE) de sementes de $V$. unguiculata tratadas com óleo essencial de $C$. winterianus, sob diferentes dosagens e aplicações.

\begin{tabular}{lccc}
\hline Método de Aplicação e Dosagens & IVE (DIAS) & VE (DIAS) & CVE (\%) \\
\hline Fumigação & & & \\
$5 \mu \mathrm{L}$ & $2,96 \mathrm{ab}$ & $6,25 \mathrm{a}$ & $15,96 \mathrm{~b}$ \\
$10 \mu \mathrm{L}$ & $2,69 \mathrm{~b}$ & $6,43 \mathrm{a}$ & $15,54 \mathrm{~b}$ \\
$15 \mu \mathrm{L}$ & $2,92 \mathrm{~b}$ & $6,28 \mathrm{a}$ & $15,92 \mathrm{~b}$ \\
$20 \mu \mathrm{L}$ & $2,67 \mathrm{~b}$ & $6,40 \mathrm{a}$ & $15,6 \mathrm{~b}$ \\
Testemunha & $3,58 \mathrm{a}$ & $5,05 \mathrm{~b}$ & $19,58 \mathrm{a}$ \\
\hline Impregnação & IVE (DIAS) & VE (DIAS) & CVE(\%) \\
\hline $5 \mu \mathrm{L}$ & $2,67 \mathrm{~b}$ & $6,3 \mathrm{a}$ & $15,92 \mathrm{~b}$ \\
$10 \mu \mathrm{L}$ & $2,63 \mathrm{~b}$ & $6,46 \mathrm{a}$ & $15,34 \mathrm{~b}$ \\
$15 \mu \mathrm{L}$ & $2,77 \mathrm{ab}$ & $6,34 \mathrm{a}$ & $15,8 \mathrm{~b}$ \\
$20 \mu \mathrm{L}$ & $2,87 \mathrm{ab}$ & $6,37 \mathrm{a}$ & $15,72 \mathrm{~b}$ \\
Testemunha & $3,61 \mathrm{a}$ & $5,04 \mathrm{~b}$ & $19,82 \mathrm{a}$ \\
\hline
\end{tabular}

*Médias seguidas de mesma letra, nas colunas, não diferem entre si significativamente pelo teste de Tukey, a 5\% de probabilidade.

\section{AGRADECIMENTO}

À Unidade Acadêmica de serra Talhada, da Universidade Federal Rural de Pernambuco, pela disponibilidade da infra-estrutura para realização da pesquisa.

Ao CNPq pelo suporte financeiro ao projeto e pela concessão de bolsas aos autores.

\section{REFERÊNCIA}

CARVALHO, N.M.; NAKAGAWA, J. Sementes: ciência, tecnologia e produção. 4.ed. Jaboticabal: Funep/Unesp. 2000.

DUDAI, N. et al. Essential oils as allelochemicals and their potential use as bioherbicides. Journal of Chemical Ecology, v.25, p.1079-89, 1999.

FURTADO, R.F. et al. Atividade larvicida de óleos essenciais contra Aedes aegypti L. (Diptera: Culicidae). Neotropical Entomology, v.34, n.5, p.843-7, 2005.

GUENTHER, E. The essential oils: individual essential oils of the plant family Graminea. New York: D. Van Nostrand Company. 1949.

GUZMÁN-MALDONADO, S.H. et al. Relationship between physical and chemical characteristics and susceptibility to Zabrotes subfasciatus (Boh.) (Coleoptera: Bruchidae) and Acanthoscelides obtectus (Say) in common bean (Phaseolus vulgaris L.) varieties. Journal Of Stored Products Research, v.32, p.53-8, 1996.

ISMAN,M.B. Botanical insecticides, deterrents, and repellents in modern agriculture and an increasingly regulated world. Annual Entomology, v.51, p.45-66, 2006. KÉITA, S.M. et al. Efficacy of essencial oil of Ocimum basilicum L. and $O$. gratissimum L. applied as an insecticidal fumigant and powder to control Callosobruchus maculates (Fab.) (Coleoptera: Bruchidae). Journal of Stored Products Research, v.37, p.339-49, 2001.

LÁSZLÓ, F. Capins na Aromaterapia: capim cidreira, limão e gengibre, palmarosa, jamrosa, citronela. Disponível em: <http://www.jardimdeflores.com.br/sinergia/ S10capins.htm>. Acessado em: 01 Mai. 2011.

LOBATO, A.K.S. et al. Ação do óleo essecial de Pipper aduncum $\mathrm{L}$. utilizado como fungicida natural do tratamento de sementes de Vigna unguiculata (L.) Walp. Revista Brasileira de Biociências, v. 5, p.915-7, 2007. LOPES, K. P. et al. Produtos naturais e fosfeto de alumínio no tratamento de sementes de feijão-macassar (Vigna unguiculata (L.) Walp.) armazenadas. Revista Brasileira de Sementes, v.22, n.2, p.109-17, 2000.

MEDEIROS, D.C. et al. Pó de folhas secas e verdes de nim sobre a qualidade das sementes de feijão caupi. Caatinga, v.20, n.2, p.94-9, 2007.

MENEZES, E.L.A. Inseticidas botânicos: seus princípios ativos, modo de ação e uso agrícola. Seropédica: Embrapa Agrobiologia, 2005. 58p. (Embrapa Agrobiologia. Documentos, 205).

OLIVEIRA, I.P.; CARVALHO, A.M. A cultura do caupi nas condições de clima e de solo dos trópicos úmidos de semi-árido do Brasil. In: ARAÚJO, J.P.P.; WATT, E.E. O caupi no Brasil. Brasília: IITA/EMBRAPA, 1988. p.63-96. ROCHA, S.F.R.; MING, L.C.; MARQUES, M.O.M. Influência de cinco temperaturas de secagem no rendimento e composição do óleo essencial de citronela Cymbopogon winterianus Jowitt. Revista Brasileira de Plantas Medicinais, v.3, p.73-8, 2000.

UVAH, I.I.; ISHAYA, A.T. Effect of some vegetable oils on 
emergence, oviposition and longevity of the bean weevil, Callosobruchus maculatus (F.). Tropical Pest Management, v.38, p.257-60,1992.
SHAAYA, E.M. et al. Plant oils as fumigants and contact insecticides for the control os stored-product insect. Journal of Stored Products Research, v.33, p. 7-15, 1997. 